\title{
Single-Crystal $\mathrm{SrTiO}_{3}$ Fiber Grown by Laser Heated Pedestal Growth Method: Influence of Ceramic Feed Rod Preparation in Fiber Quality
}

\author{
D. Reyes Ardila, M.R.B. Andreeta, S.L. Cuffini, A.C. Hernandes, \\ J.P. Andreeta, Y.P. Mascarenhas \\ Grupo de Crescimento de Cristais, \\ Instituto de Física de São Carlos, \\ Universidade de São Paulo, \\ C.P. 369, 13560-970 São Carlos - SP, Brazil; \\ e-mail: hernandes@ifsc.sc.usp.br
}

Received: February 2, 1998; Revised: June 25, 1998

\begin{abstract}
The rapidly spreading use of optical fiber as a transmission medium has created an interest in fiber-compatible optical devices and methods for growing them, such as the Laser Heated Pedestal Growth (LHPG). This paper reports on the influence of the ceramic feed rod treatment on fiber quality and optimization of ceramic pedestal processing that allows improvements to be made on the final quality in a simple manner. Using the LHPG technique, transparent crack-free colorless single crystal fibers of $\mathrm{SrTiO}_{3}(0.50 \mathrm{~mm}$ in diameter and 30-40 $\mathrm{mm}$ in length) were grown directly from green-body feed rods, without using external oxygen atmosphere.
\end{abstract}

Keywords: laser heated pedestal growth, $\mathrm{SrTiO}_{3}$, crystal growth

\section{Introduction}

Due to the increasing use of optical fibers in telecommunication networks, there is a need for fiber geometry that is compatible with optical devices. The development of a faster process to obtain high quality single crystal fibers from ceramic pedestal rods is therefore considered desirable. The $\mathrm{SrTiO}_{3}$ compound was used to study different stages in this process systematically.

Pure $\mathrm{SrTiO}_{3}$ is colorless, transparent in the visible region, and is an insulator (band gap: $3.2 \mathrm{eV}$ ) when it contains stoichiometric amounts of oxygen. It presents some interesting chromic behavior, which is thermally activated and depends markedly on the annealing temperature and on the quenching rate ${ }^{1,2}$. The oxygen vacancies produce a wide range of colors, from yellow or light brown ${ }^{3}$ to blue-violet or black $\mathrm{k}^{4-7}$. This effect is called the thermochromism of $\mathrm{SrTiO}_{3}$ and it can be completely reversed by reheating in an oxygen atmosphere or cooling slowly to room temperature from any temperature above $850{ }^{\circ} \mathrm{C}^{1,2}$. Furthermore, even very small amounts of oxygen vacancies can produce these color changes. In fact, in yellow or light brown samples, it was very difficult to detect changes of the oxygen vacancies by usual thermogravimetric analysis because the weight loss is too small ${ }^{4}$. It is important to note that the effect of oxygen vacancies was not negligible, since they caused large modifications in the sample's properties, mainly in their electronic transport and optical absorption ${ }^{2,7,8}$.

$\mathrm{SrTiO}_{2.98}$ or $\mathrm{SrTiO}_{3-\mathrm{x}}$ with $\mathrm{x}=0.28,0.17$ and 0.08 presented a dark blue-violet colored, n-type metallic conductivity $^{5,6}$. Further studies demonstrated that conductivity and optical absorption exhibited a proportional relationship; from a general point of view, the bluish colored samples indicate metallic conductivity and optical absorption in the infrared range ${ }^{2,5,6}$, while the slightly reduced ones showed absorption in the visible range and, usually, lower values of conductivity ${ }^{2,7}$.

In all the above-mentioned cases, it is very important to prepare the conditions carefully and to characterize a $\mathrm{SrTiO}_{3}$ sample as well as possible before studying any of its properties. 
A surprisingly wide variety of crystal growth techniques that have been used for $\mathrm{SrTiO}_{3}$ were reviewed by K. Nassau and A.E. Miller ${ }^{9}$. The solution growth has proved to produce much more perfect crystals than the Verneuil growth ${ }^{10}$, however, neither the solution growth technique nor the other meltgrowth techniques reached the point where $\mathrm{SrTiO}_{3}$ crystals can be grown with stoichiometric amounts of oxygen under reasonably controlled conditions. Currently, only the Verneuil product is available commercially?.

Table 1, which summarizes the growth conditions of the most important techniques, shows that the titanate single crystals presented dark colors in most cases, indicating that it is necessary to use an oxygen atmosphere to obtain colorless crystals.

This paper reports on a systematical study of the influence of the feed rod characteristics (geometry, thermal treatments, and density) on the quality of final crystal fiber. Thus, we have found ceramic processing conditions that permit the growth of stoichiometric $\mathrm{SrTiO}_{3}$ single crystal fiber without using external oxygen atmosphere.

\section{Experimental Procedure}

\section{A. Ceramic pedestal preparation}

Several ceramic pedestals were prepared under different conditions in order to compare their performance as ceramic feed and seed rods for fiber growth.

\section{A-1. Synthesis}

$\mathrm{SrTiO}_{3}$ powder was prepared from a solid state reaction of high purity $\mathrm{SrCO}_{3}\left(99.999 \%\right.$, Merck) with $\mathrm{TiO}_{2}$ (99.999\%, Merck). Appropriate molar ratios were mixed and ground in an agate mortar and then calcinated several times at $1000{ }^{\circ} \mathrm{C}$ for $12 \mathrm{~h}$. The cooling rate of the furnace was not controlled. The solid state reaction product was measured in each calcination step using X-ray diffraction analysis. This process was repeated until the $\mathrm{SrTiO}_{3}$ pure phase was reached. The average particle size of the final powder was around $0.30 \mu \mathrm{m}$.

\section{A-2. Square Section Pedestals}

$\mathrm{SrTiO}_{3}$ powder was uniaxially pressed into cylindrical pellets under $1.5 \mathrm{KPa}$ and then isostatically pressed at 100 $\mathrm{MPa}$. In some cases, polyvinyl alcohol (PVAl) was added to the powder, as a binder, in order to improve its compactness (see Table 2). The pressed pellets were sintered at different temperatures. Density data were obtained using the Archimedes method.

The ceramic pellets were polished and cut into 700-800 $\mu \mathrm{m}$ square section rods using a diamond inner hollow saw (Microslice 4 Malvern). In the last step of pedestal preparation, the larger rods $(3 \mathrm{~cm}$ in length) were annealed in an oxygen atmosphere at $1000{ }^{\circ} \mathrm{C}$ to release them from stress and to avoid oxygen vacancies. These pedestals were used as feed and seed rods in the crystal growth process.

\section{A-3. Circular section pedestals}

The circular rods were obtained by the extrusion process. This process consists of shaping an object by pushing a material through an opening of predefined shape. A wet mixture of powder and binder was used to carry out the extrusion into rods through a stainless-steel die with a $1 \mathrm{~mm}$ diameter orifice. The size and shape of our extrusion ${ }^{11}$ machine allows us to obtain circular rods of $1 \mathrm{~mm}$ in diameter and more than $10 \mathrm{~cm}$ in length. The extruded green-rods were air-dried to avoid shrinkage. Table 2

Table 1. Titanates crystal growth conditions with conventionally methods.

\begin{tabular}{|c|c|c|c|c|c|c|}
\hline \multirow[b]{2}{*}{ Methods } & \multicolumn{4}{|c|}{ Rod powder preparation } & \multicolumn{2}{|c|}{ Growth parameters } \\
\hline & $\begin{array}{c}\text { Syntesis of } \\
\text { starting materials }\end{array}$ & $\begin{array}{l}\text { Rod dimensions } \\
\text { (diameter- } \mathrm{mm} \text { ) }\end{array}$ & $\begin{array}{c}\text { Sinterization } \\
\text { conditions }\end{array}$ & Rate $(\mathrm{mm} / \mathrm{min})$ & Atmosphere & $\begin{array}{l}\text { Single-crystal } \\
\text { color }\end{array}$ \\
\hline $\begin{array}{l}\text { Verneuil flame- } \\
\text { fusion growth }\end{array}$ & & & & & oxygen & colorless \\
\hline Flux growth ${ }^{10^{*}}$ & & & & & air & $\begin{array}{l}\text { brown/light } \\
\text { brown }\end{array}$ \\
\hline $\begin{array}{l}\text { Radio-frequency } \\
\text { induction }^{6^{*}}\end{array}$ & $1200^{\circ} \mathrm{C}, 3$ days & & & & vacuum/air & dark blue-violet \\
\hline $\begin{array}{l}\text { Floating zone } \\
\text { growth }^{5^{*}}\end{array}$ & $\begin{array}{c}1000{ }^{\circ} \mathrm{C} 10 \mathrm{~h} \text { in } \\
\text { air }\end{array}$ & 10 & $1300^{\circ} \mathrm{C}, 3 \mathrm{~h}$ & 0.16 & air & black \\
\hline 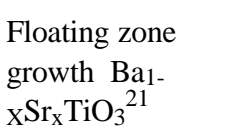 & $\begin{array}{c}1000{ }^{\circ} \mathrm{C} 10 \mathrm{~h} \\
\text { in air }\end{array}$ & $5-10$ & none & $0.016-0.033$ & oxigen & yellow \\
\hline $\mathrm{LHPG} \mathrm{BaTiO}_{3}{ }^{20}$ & & $1-2$ & $\begin{array}{c}1400{ }^{\circ} \mathrm{C}, 6 \mathrm{~h} \\
\text { oxigen }\end{array}$ & $5-15$ & air & blue \\
\hline
\end{tabular}

References with * correspond to $\mathrm{SrTiO}_{3}$. 
shows the conditions and powder composition used in the preparation process. The pedestals from batch $\mathrm{D}$ (see Table 2) were sintered at $1400{ }^{\circ} \mathrm{C}$ for $6 \mathrm{~h}$, while the ones from batch $\mathrm{E}$ and $\mathrm{F}$ were not thermally treated either to remove the binder or to improve densification. Materials with the same characteristics were used as feed and seed rods in the fiber growing process.

\section{B. Laser Heated Pedestal Growth (LHPG) method}

The LHPG method has been shown to be a powerful method for rapidly growing small diameter single crystals, particularly oxides of high melting temperature, for both properties studies and fiber devices ${ }^{12,13}$.

The LHPG equipment used in this investigation consisted of a power source $\left(\mathrm{CO}_{2} 100 \mathrm{~W}\right.$ laser$)$, an optical layout and a growth chamber. To minimize the temperature oscillations in the molten zone, an additional control system was applied, in which a photodetector signal proportional to the intensity of the light coming from the melting zone was used to feed back the $\mathrm{CO}_{2}$ laser power. The growing fiber and the melting zone were observed through a monocular microscope ${ }^{14}$.

Pulling and feeding rates were between 0.15-0.60 $\mathrm{mm} / \mathrm{min}$ and $0.05-0.60 \mathrm{~mm} / \mathrm{min}$ respectively, using square section rods (length: $30 \mathrm{~mm}$, cross section size: 0.8-1.0 $\mathrm{mm}$ ). When using extruded circular section rods (length: $30-50 \mathrm{~mm}$ cross section diameter: $1.0 \mathrm{~mm}$ ), the same parameters used were between $0.30-0.50 \mathrm{~mm} / \mathrm{min}$ and 0.10 $0.20 \mathrm{~mm} / \mathrm{min}$, respectively. Additional details can be found in Ref. 14.

\section{Ceramic pedestals and fibers characterization}

Powder diffraction data were collected using a RigakuDenki diffractometer with $\mathrm{Cu} \mathrm{K}_{\alpha}$ radiation supplied by a rotating anode source. X-ray Laue reflection was used to analyze the single-crystal fibers. Their crystallographic parameters were obtained using a CAD-4 four-circle auto- matic diffractometer (Enraf Nonius). The microstructure was analyzed with a digital scanning electron microscope (Zeiss DSM 960). The conventional two-probe method was used to measure fiber resistivity.

\section{Results and Discussion}

Fiber growth, using square section pedestals, presented low stability of the melting zone shape, mainly with the lower density feed rods. Table 2 indicates that the batch A pedestals presented the lowest density. Pedestal densification improved when sinterization was carried out at higher temperatures. Thus, the pedestals from batch $\mathrm{B}$ and $\mathrm{C}$ were denser and presented a grain growth, when compared to A, as shown in Fig. 1.

The fiber growth process, using rods from batch A, showed a considerable variation in diameter. Fibers showing a more regular diameter were obtained, with very low diameter variations, by increasing pedestal densification (batch B and C). However, they presented inclusions and cracks.

Typically, fibers grown from batch A were yellowish, while those grown from batch $\mathrm{B}$ and $\mathrm{C}$ were black and brown, respectively. Therefore, fiber color was directly related to the pedestal thermal treatment and, since stoichiometric $\mathrm{SrTiO}_{3}$ is colorless, it can be concluded that higher sintering temperatures yield darker fibers, and that all are oxygen deficient. The best results, when using square section pedestals, were obtained only by re-pulling primary fibers. Although the final fibers grown with this procedure were single-crystals with a regular diameter, they presented a yellowish color ${ }^{15}$.

The use of square section pedestals presented some disadvantages, mainly due to the mismatch between the fiber cross section and the laser beam geometries (square and circular, respectively). This mismatch does not allow the laser beam to melt the pedestal efficiently, leaving the square corners solid and affecting fiber growth. The circu-

Table 2. Preparation and macroscopic characteristics of $\mathrm{SrTiO}_{3}$ ceramic pedestals.

\begin{tabular}{|c|c|c|c|c|c|c|}
\hline \multirow[b]{2}{*}{ Samples } & \multicolumn{3}{|c|}{ Square section pedestals } & \multicolumn{3}{|c|}{ Circular section pedestals } \\
\hline & A & $\mathrm{B}$ & $\mathrm{C}$ & $\mathrm{D}$ & $\mathrm{E}$ & $\mathrm{F}$ \\
\hline $\begin{array}{l}\text { Powder composi- } \\
\text { tion }\end{array}$ & $\mathrm{SrTiO}_{3}$ & $\mathrm{SrTiO}_{3}$ & $\mathrm{SrTiO}_{3}$ & $\mathrm{SrTiO}_{3}$ & $\mathrm{SrTiO}_{3}$ & $\mathrm{SrCO}_{3}+\mathrm{TiO}_{2}$ \\
\hline Binder agent & PVA & none & PVA & PVA & PVA & PVA \\
\hline Pressing & \multicolumn{3}{|c|}{ uniaxial (1.5 KPa) isostatic (100 MPa) } & & & \\
\hline Sinterization & $1200^{\circ} \mathrm{C}, 24 \mathrm{~h}$ & $1600{ }^{\circ} \mathrm{C}, 5 \mathrm{~h}$ & $1400{ }^{\circ} \mathrm{C}, 6 \mathrm{~h}$ & $1400^{\circ} \mathrm{C}, 6 \mathrm{~h}$ & none & none \\
\hline Density $\left(\mathrm{g} / \mathrm{cm}^{3}\right)$ & 3.250 & 4.200 & 4.800 & 4.816 & 2.087 & 2.335 \\
\hline $\begin{array}{l}\% \text { Theoretical } \\
\text { density }\end{array}$ & 62 & 82 & 94 & 94 & 41 & - \\
\hline color & white yellow & dark gray & gray & gray & white yellow & white \\
\hline
\end{tabular}


lar section pedestals prepared by the extrusion process allow matching up of the feed rod geometry with the laser beam. It is important to note that a variety of green-body defects can appear during the extrusion process. These defects includes: lamination, surface and edge tearing, bridge and core cracking, column splitting, segregation and preferred orientation of particles ${ }^{11}$. However, the quality of the extruded bodies is highly influenced by several factors and depends mainly on each material. Table 2 shows in detail the different circular section pedestal conditions in which the bodies were prepared. In the sintering process, the pedestals from batch D presented the best densification and reached the same density values as isopressed rods (batch $\mathrm{C}$ ). Moreover, an analysis of their microstructure showed similar grain sizes, as shown in Fig. 1b. Note that the green-rod densities from batch $\mathrm{E}$ to $\mathrm{F}$ were almost the same and were used as green extruded rods without any temperature treatment to burn out the binder or for the sintering process. Moreover, the binder burnout stage during the crystal growth process did not cause any problems. Typically, during the growth, a dark band appeared immediately below the molten zone, showing the residual carbon
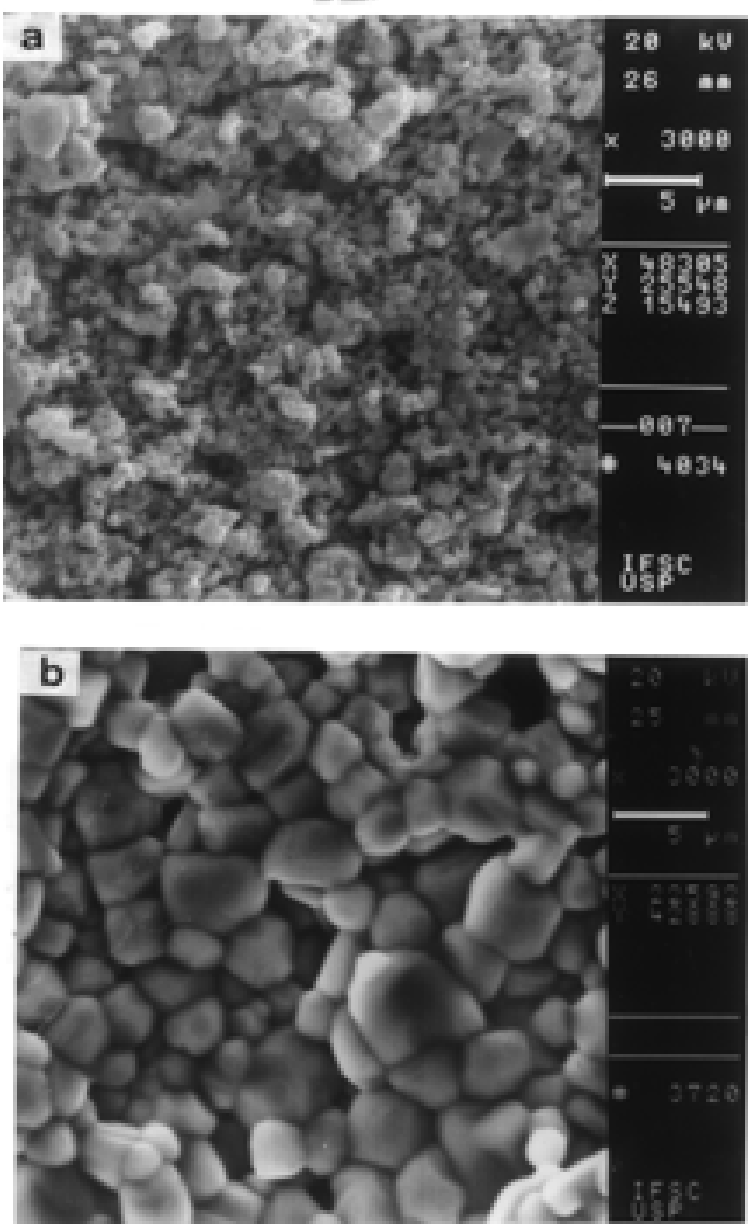

Figure 1. SEM microphotographs of the grain growth of square section pedestals from batches $\mathrm{A}$ (a) and $\mathrm{C}$ (b). compound caused by combustion of the organic binder. Figure 2, the SEM microphotographs of pedestals from batch $\mathrm{E}\left(\mathrm{SrTiO}_{3}\right)$ and batch $\mathrm{F}\left(\mathrm{SrCO}_{3}-\mathrm{TiO}_{2}\right)$, show a different distribution of grain size, indicating an evident mixture of two phases in Fig. 2b. In the latter, the large crystals with regular shapes correspond to $\mathrm{TiO}_{2}$ while the smaller ones, with poorly defined shapes, correspond to $\mathrm{SrCO}_{3}$. The degree of densification in both cases was very low.

Figure 3 shows the first-grown fiber drawn using the feed rods from batches D, E and F. Although all the fibers grown in highly stable conditions displayed a regular diameter, observation shows that the fiber from batch D presented inclusions and cracks with low crystal quality. The fibers from batches $\mathrm{E}$ and $\mathrm{F}$, on the other hand, were totally free of these types of defects. The most surprising result was obtained using the feed rods from batch $\mathrm{F}$, where a crack-free, transparent and colorless fiber was obtained in the first pulling process. The fiber was about $0.50 \mathrm{~mm}$ in diameter and $30 \mathrm{~mm}$ in length.
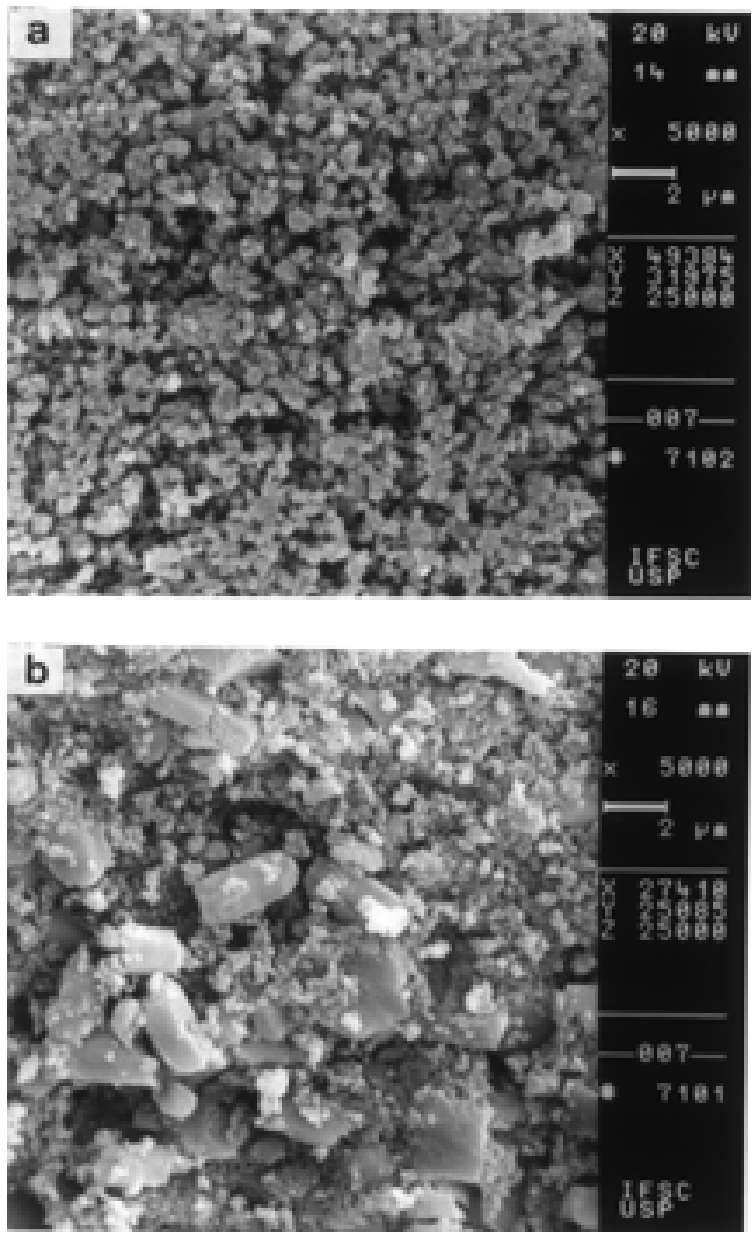

Figure 2. SEM microphotographs of the grain distribution in circular section pedestals (a) from batch $\mathrm{E}\left(\mathrm{SrTiO}_{3}\right)$ and (b) from batch $\mathrm{F}\left(\mathrm{SrCO}_{3}\right.$ $-\mathrm{TiO}_{2}$ ). 


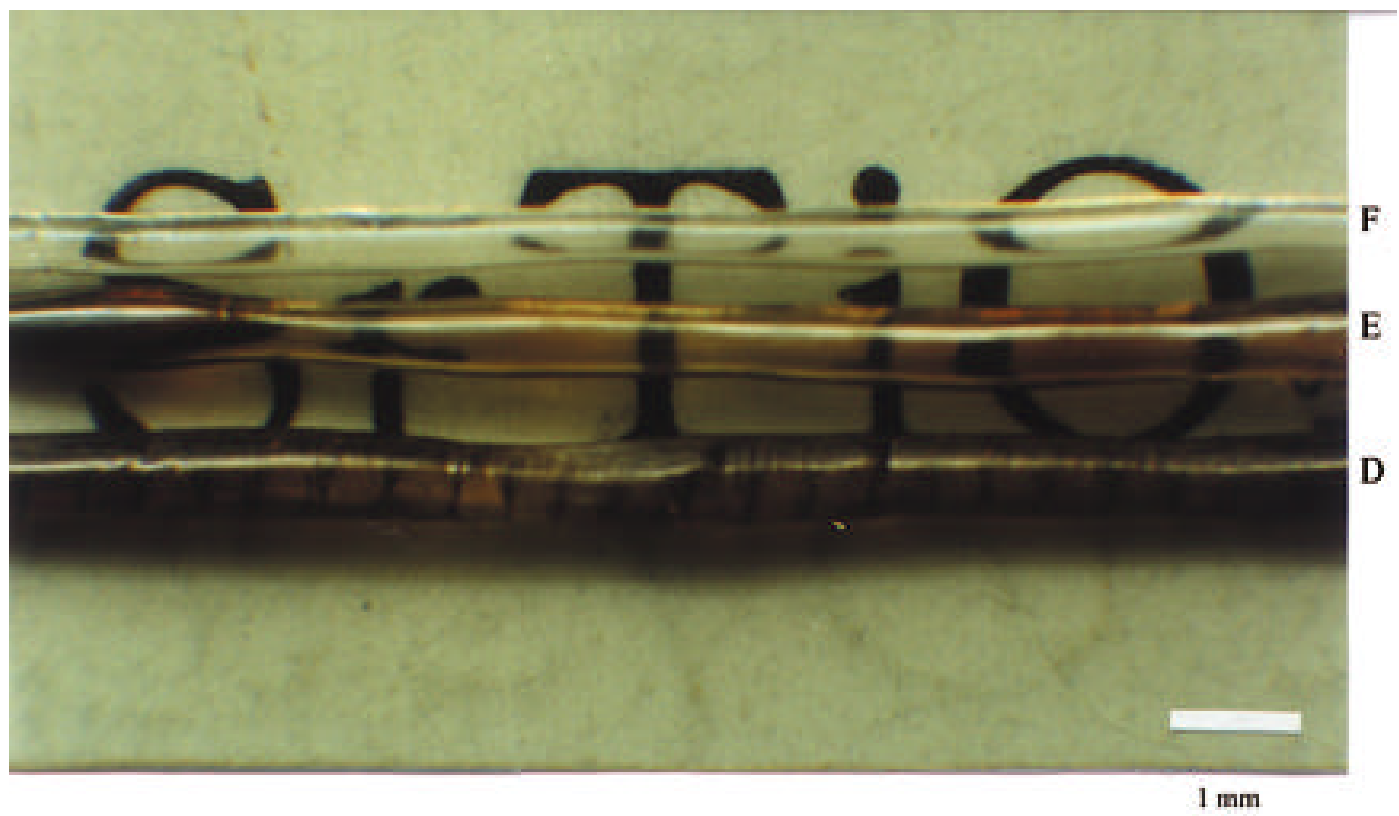

Figure 3. Photographs of the fibers drawn directly from the circular section pedestals, using ceramic feed rods from batch D, E and F, whose diameter sizes were around $0.70 \mathrm{~mm}, 0.60 \mathrm{~mm}$ and $0.50 \mathrm{~mm}$, respectively.

The inclusion of $\mathrm{CO}_{2}$ gas presented no problem during growth. Furthermore, the thermal dissociation of $\mathrm{CO}_{2}$ at high temperatures $(2300 \mathrm{~K}-3400 \mathrm{~K})$ produced a local oxidation atmosphere with high reactivity owing to the $\mathrm{O}$ atom formation during dissociation ${ }^{16}$. Thus, the decomposition of $\mathrm{SrCO}_{3}$ in batch $\mathrm{F}$ produced a local pressure of $\mathrm{CO}_{2}$ and the dissociation of $\mathrm{CO}_{2}$, at melting temperature, caused a local oxidation atmosphere. Loss of oxygen was thus depressed and transparent-colorless fibers (batch F) were obtained.

Figure 4 shows that the microstructure of the molten zone of the feed rod from batch $\mathrm{F}$ exhibited grain sizes similar to those of the densest isopressed rods from batch C (see Fig. 1b). Although the microstructure in the same region of the rod from batch $\mathrm{D}$ displayed higher densification, the final quality of crystal fiber was inferior. For this reason, we found in this case that it is needless to carry out a previous sintering process since it does not improve growth conditions but worsens the quality of crystal fiber.

The batch $\mathrm{F}$ fibers were crushed, ground and analyzed by X-ray powder diffraction. The X-ray diffraction pattern did not reveal the presence of any segregated phase, from which it can be concluded that the starting materials $\left(\mathrm{SrCO}_{3}\right.$ - $\mathrm{TiO}_{2}$ ) underwent a complete reaction that resulted in pure $\mathrm{SrTiO}_{3}$. Figure 5 shows a Laue photograph of this single crystal fiber, with the X-ray beam in a direction perpendicular to the growth axis. The preferential growth orientation was [110] with cubic crystal lattice, $a=3.905(1) \AA$.

As mentioned before, the batch $\mathrm{F}$ fibers were transparent and colorless, so that they must contain stoichiometric amounts of oxygen. Although the other fibers, from batch $\mathrm{E}$, were also crack-free they were yellowish, revealing the
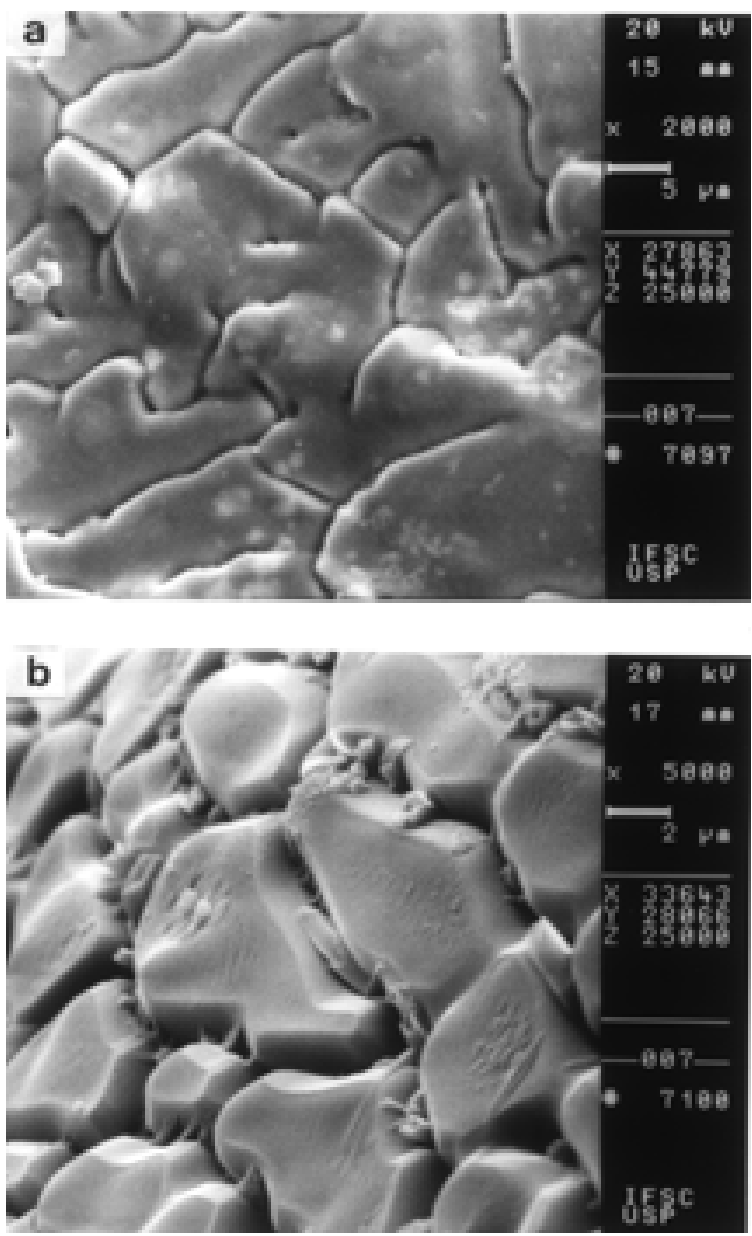

Figure 4. SEM microphotographs of the molten zone obtained, using feed rods from batch $\mathrm{D}$ (a) and $\mathrm{F}(\mathrm{b})$. 


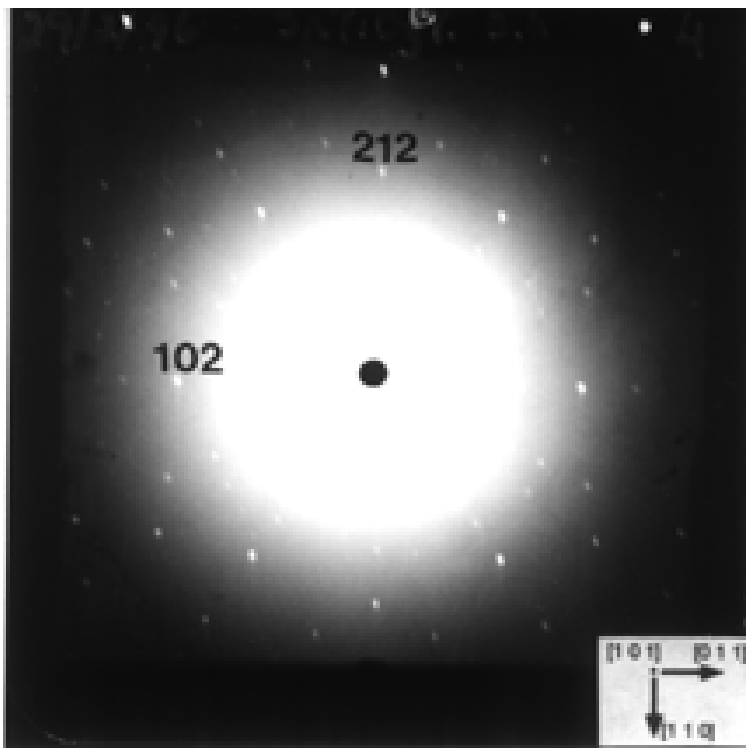

Figure 5. Laue photograph of an $\mathrm{SrTiO} 3$ single crystal fiber with the $\mathrm{X}$-ray beam perpendicular to growth direction.

presence of oxygen vacancies. This observation was confirmed by resistivity measurements, as shown in Fig. 6. The transparent and colorless fibers (curve a) presented higher resistivities in the whole temperature range and a roomtemperature band gap of $3.2 \mathrm{eV}$. These results are in very good agreement with the published data of pure $\mathrm{SrTiO}_{3}$ when it contains stoichiometric amounts of oxygen. Curve $\mathrm{b}$, in the same figure, shows a decrease in resistivity values in the same temperature range with respect to the latter fibers, indicating the presence of oxygen vacancies. It was observed that the loss of oxygen produced decreased resistivity and a change in the color of the samples. Several authors have reported resistivities of around 1-20 $\Omega \mathrm{cm}^{7,17}$ at room temperature in samples with slightly reduced, and values of around $10^{-1}-10^{-3} \Omega \mathrm{cm}^{5,6,18}$ for samples with more reduced strontium titanate. In every case, after thermal treatment in the oxygen atmosphere, the yellow, blue or dark blue samples became colorless, and their resistivities were above $10^{9} \Omega \mathrm{cm}$. The samples showed semiconductor to metallic-like behavior, depending on their levels of reduction.

The advantages of the fiber growth procedure used in batch $\mathrm{F}$ must be emphasized, since the preparation process was greatly simplified. In this case, when the pedestal was pushed toward the molten zone, binder burnout (PVAl), decomposition, reaction of starting materials, sinterization of the compound and the growth of a stable single-crystal $\mathrm{SrTiO}_{3}$ fiber were carried out in one operation, thus reducing the time and cost of preparation.

\section{Conclusions}

$\mathrm{SrTiO}_{3}$ single crystal fibers were successfully grown directly from green-body feed rods by LHPG. These crystal

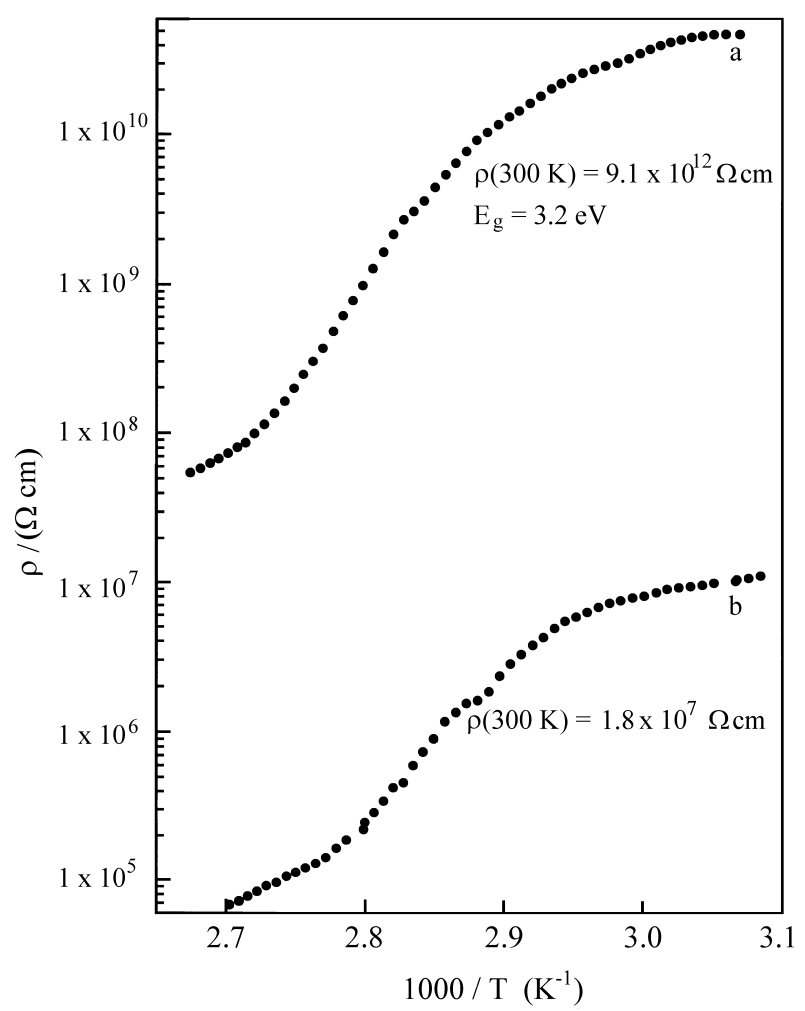

Figure 6. Resistivity as a function of temperature of $\mathrm{SrTiO}_{3}$ fibers. Curve a: $\mathrm{SrTiO}_{3}$ fibers from batch $\mathrm{F}$ (colorless). Curve b: $\mathrm{SrTiO}_{3}$ fibers from batch E (yellow).

fibers were transparent and colorless, and no external oxygen atmosphere was used during crystal growth or by high-temperature annealing after growth.

Dark-colored crystals grown by conventional growth techniques presented non-stoichiometry, which occurs due to feed-rod-oxygen loss during the synthesis and sinterization process. Furthermore, these methods present larger surface-to-volume ratio of melt than LHPG does. For this reason, the oxygen loss may be depressed during the LHPG process, which minimizes this effect.

Primary fibers, grown directly from feed rods, demonstrated a high dependence on the feed rod geometry. The growth was highly stable when using cylindrical section feed rods, as compared to what occurred when using the squared section rods. In the first case, the melting zone volume was maintained constant, which lead to regular diameter fibers. The laser beam diameter was adjusted to the feed rod geometry, thus avoiding the formation of corners and their periodic falls that produce very unstable growth.

Different preparation conditions of the cylindrical section feed rods showed that treating them at high temperatures worsened the quality of the final fiber, even though the feed rods presented a high density. The best quality fiber was grown directly from green-body feed rod, without any thermal treatment. Therefore, the sintering process of the 
feed rods did not improve any crystal growth conditions, as had been expected. Indeed, it is a known fact that most of materials were grown as high quality fibers when sintered feed rods were used ${ }^{19-21}$. Furthermore, there are few compounds that presented a behavior similar to that of $\mathrm{SrTiO}_{3}$ with respect to the influence of feed rod density on fiber quality, i.e. alumina ${ }^{22}$.

Additionally, it was demonstrated that the synthesis of the starting materials before the growth process by LHPG did not improve any of its conditions but, on the contrary, it lead to larger amounts of oxygen vacancies. It is possible that, if cooling had been carried out slowly from $1000{ }^{\circ} \mathrm{C}$ to $850{ }^{\circ} \mathrm{C}$ (the "freezing-in" temperature for the diffusion of oxygen in $\mathrm{SrTiO}_{3}$ ) during synthesis, the $\mathrm{SrTiO}_{3}$ powder would have been stoichiometric. Nevertheless, it seems better to bypass this previous synthesis stage.

A comparison of the different batches of feed rods (A to $\mathrm{F}$ ) shows that grain size, size distribution and the green density did not significantly affect the quality of the crystal. In the case of batch $\mathrm{F}$, the best conditions for growing the high quality stoichiometric $\mathrm{SrTiO}_{3}$ single crystal fibers were found to be by LHPG, using a faster and simpler procedure. It must be pointed out that the cylindrical geometry of the feed rods and a local oxidation atmosphere due to a partial dissociation of $\mathrm{CO}_{2}$ are the most important contributions.

\section{Acknowledgments}

The financial support of CNPq, FAPESP and FINEP is gratefully acknowledged. The authors thank Prof. Dr. J.A. Eiras for putting his laboratory's furnaces and electrical measurement equipment at their disposal, and G. Catarino and J.A. Lopez da Rocha for their technical assistance in the X-ray analysis. S.L. Cuffini thanks CNPq, M.R.B. Andreeta thanks FAPESP and D. Reyes Ardila thanks CAPES for the fellowship grants received from those institutions.

\section{References}

1. Wild, R.L.; Rockar, E.M.; Smith, J.C. Phys. Rev. B, v. 8, n. 8, p. 3828-3835, 1973.

2. Gandy, H.W. Phys. Rev., v. 113, n. 3, p. 795-800, 1959.

3. Howard, S.A.; Yau, J.K.; Anderson, H.U. J. Appl. Phys., v. 65, n. 4, p. 1492-1498, 1989.

4. Paladino, A.E.; Rubin, L.G.; Waugh, J.S. J. Phys. Chem. Solids, v. 26, p. 391-397, 1965.
5. Higuchi, M.; Aizawa, K.; Yamaya and Kodaira,, K. J. Solid State Chem., v. 92, p. 573-577, 1991.

6. Gong, W.; Yun, H.; Ning, Y.B.; Greedan, J.E.; Datars, W.R.; Stager, C.V. J. Solid State Chem., v. 90, p. 320330, 1991.

7.Lee, C.; Yahia, J.; Brebner, J.L. Phys. Rev. B, v. 3 n. 8, p. 2525-2533, 1971.

8. Yamada, H.; Miller, G.R. J. Solid State Chem., v. 6, p. 169-177, 1973.

9. Nassau, K.; Miller, A.E. J. Crystal Growth, v. 91, p. 373-381, 1988 and references therein.

10. Sheel, H.J.; Bednorz, J.G.; Dill, P. Ferroelectrics, v. 13, p. $507,1976$.

11. Ceramic Processing Before Firing. Edited by Onoda Jr., G.Y.; Hench, L.L. John Wiley and Sons, New York, 1978). Chapter 29, p, 391.

12. Haggerty, J.S.; Menashi, W.P.; Wenckus, J.F. Methods for Forming Refractory Fibers by Laser Energy, U.S. Patent 3944640, March 16, 1976; Apparatus for Forming Refractory Fibers, U.S. Patent 4012213, March 15, 1977.

13. Feigelson, R.S. Crystal Growth of Electronics Materials, North-Holland, Amsterm, p. 127, 1985.

14. Profokiev, V.V.; Andreeta, J.P.; de Lima, C.J.; Andreeta, M.R.B.; Hernandes, A.C.; Carvalho, J.F.; Kamshilin, A.A. Jääskeläinen, T. J. Crystal Growth, vol. 137, p. 528-534, 1994.

15. Reyes Ardila, D.; Andreeta, M.R.B.; Cuffini, S.L.; Hernandes, A.C.; Andreeta, J.P.; Mascarenhas, Y.P. Ferroelectrics, v. 186, p. 141-144, 1996.

16. Fujii, N.; Sagawai, S.; Sato, T.; Nosaka, Y.; Miyama, H. J. Phys. Chem., v. 93, p. 5474-5478, 1989.

17. Lee, C.; Destry, J.; Brebner, J.L. Phys. Rev. B, v. 11, n. 6, p. 2299-2310, 1975.

18. Frederikse, H.P.R.; Thurber, W.R.; Hosler, W.R. Phys. Rev. A, v. 134, p. 442-445, 1964.

19. Guo, Ruyan; Bhalla, A.S.; Sheen, Jyh; Ainger, F.W.; Erdei, S.; Subbarao, E.C.; Cross, L.E. J. Mater. Res., v. 10, n. 1, p. 18-25, 1995.

20.Saifi, M.; Dubois, B.; Vogel, E.M.; Thiel, F.A. J. Mater. Res., v. 1, n. 3, p. 452-456, 1986.

21. Wilde, J.P.; Jundt, D.H.; Galambos, L.; Hesselink, L. J. Crystal Growth, v. 114, p. 500-506, 1991.

22. Piramoon, M.R.; Neeson, C.; Troadec, T.; Wang, J.; Ponton, C.B.; Marquis, P.M. British Ceramics Proceedings, Institute of Materials, p. 135-146, 1993. 